\title{
Sobre la actualidad de Fichte y su idea de una educación para la libertad
}

\author{
About the topicality of Fichte and his idea of an education for \\ freedom
}

\author{
Virginia López-Domínguez \\ yotrascendental@hotmail.com \\ (Universidad Complutense, Madrid, España)
}

\begin{abstract}
Resumen: Dado que Fichte prefirió evitar las revoluciones violentas y abogó por las transformaciones sociales progresivas, su sistema de filosofía desembocó en una educación para la libertad, cuyo objetivo era formar ciudadanos responsables, libres y creativos, comprometidos con la verdad. En este artículo se muestran cuáles son los fundamentos de esa educación, ya en el período de Jena, para centrarse en los principios desarrollados por él durante su época de Berlín (preferentemente en los Aforismos sobre educación o en La esencia del sabio), además de confrontarlos con la situación pedagógica actual en escuelas y Universidades.
\end{abstract}

Palabras clave: Fichte; Educación; Libertad; Sabio; Universidad.
Abstract: As Fichte preferred to avoid violent revolutions and defended progressive social transformations, his system of Philosophy resulted in an education for freedom, which goal was to form responsible, free and creative citizens, committed to the truth. This paper shows which are the foundations of that education, already in the times of Jena, focuses on the principles developed by him during the maturity period (particularly in the Aphorisms on education or The essence of the Scholar), and confronts them with the actual pedagogic situation at schools and Universities.

Keywords: Fichte; Education; Freedom; Scholar; University.

Referirme a la educación en Fichte me ha permitido volver a una cuestión en la que no reparaba desde hacía treinta y cuatro años, ya que uno de los últimos capítulos de mi Tesis doctoral sobre él (defendida en 1985) se tituló justamente "El mesiazgo de la educación”. Nunca lo publiqué y, por supuesto, en todos estos años apenas se trató el tema en foros públicos, prácticamente hasta la reedición en 2017 del libro de Ernst Bergmann Fichte, Erzieher zum Deutschtum, donde lo que destaca y discute es la idea de una educación nacional en el marco de una teoría general de la educación. Y uno no puede dejar de pensar por qué ocurrió este sintomático olvido, dado que la exigencia de una nueva pedagogía acorde a los modelos sociopolíticos propuestos por Fichte es una de las consecuencias naturales de su filosofía. En lo que a mí se refiere, tengo que confesar que no me ocupé de la educación porque creía que no le iba a interesar a nadie. Por entonces, los especialistas estaban más preocupados por continuar la edición crítica de las obras del idealista y ver la compatibilidad entre 
las distintas versiones de la Wissenschafstlehre. Pero lo curioso del caso es que, mientras tanto, durante ese largo tiempo, se fueron remodelando todos los sistemas educativos españoles y europeos. Y lo hicieron siguiendo el modelo norteamericano, de acuerdo con las exigencias del gran mercado capitalista, a estas alturas ya globalizado. Eso precisamente hace pensar que quizás la falta de atención a los planteamientos pedagógicos de Fichte no fuera arbitraria. Más bien parece que no se lo hubiese querido escuchar, porque, si hay algo que define al idealismo ético es que pretende constituir -según dijo el mismo Fichte - el primer sistema de la libertad ${ }^{1}$ y, como resultado, la educación que deriva de él no puede ser sino una "educación para la libertad", lo cual contradice completamente el espíritu educativo impuesto ya en nuestros días, cuyo objetivo parece ser la proliferación de individuos esclavizados por los medios de comunicación y las grandes empresas que los sostienen, más atentas a desarrollar unas habilidades específicas en los educandos que les sean útiles en aquellos campos del saber aplicado y que, además, ellas quieran promocionar en el mercado. De este modo, de principio a fin, la educación se ha convertido hoy en un negocio, en una transacción externa, puramente material. E insisto: esto se opone de modo flagrante a la visión idealista.

Indudablemente, la cuestión de la educación siempre esconde un trasfondo político porque la sociedad forma a sus miembros con el fin de perdurar y reproducirse a sí misma, acoplándolos a sus propios fines que, por supuesto, no son los generales sino los de los grupos dominantes. Como es bien sabido, Fichte fue un defensor de la revolución francesa, de un orden político diferente al que existía en ese momento en Alemania, pero, ya desde su escrito de 1793 sobre la reivindicación de la libertad de pensar, ${ }^{2}$ desaconsejó las transformaciones sociales abruptas y violentas por el temor a la posible involución de las revoluciones políticas, que, después de todo, no son sino transformaciones externas que no afectan moralmente al individuo $\mathrm{y}$, en consecuencia, no tienen la fuerza de la convicción, de la exigencia interior. Esta transformación gradual de la sociedad sólo podría realizarse con paso firme y seguro, sin posibilidad de reversión, cuando es reflejo de una metamorfosis interna, la cual puede ser incitada sólo a través de la educación. Fichte fue muy pronto consciente de la necesidad de una reforma educativa como preparación para el cambio social. Según Willy Kabitz, ${ }^{3}$ el origen de esta idea en él se remonta a antes de 1788, a la lectura o conocimiento de Leibniz, Rousseau, Lessing, Pestalozzi o Christian Gotthilf

1 Carta de Fichte a Baggesen de 1795, GA III, 2, № 282 b, p. 300. Véase asimismo el esbozo de esta carta en Sch. 231, I, pp. 449 s./ GA III, 2, N 282 b, p. 298.

2 Zurückforderung der Denkfreiheit von den Fürsten Europens, die sie bisher unterdrückten, GA I, 1, p. 169.

3 Studien zur Entwicklungsgeschichte der Fichteschen Wissenschaftslehre aus der Kantischen Philosophie (Berlin: Reuther \& Reichard, 1902. Reimpresión: Darmstadt: Wissenschatliche Buchgesellschaft, 1968), pp. $8 \mathrm{~s}$. Ver asimismo Léon, X. (1922-1927). Fichte et son temps. 3 vols. Paris: Armand Colin; reedición 1954-59) I, pp. 62-66. 
Salzmann (1744 - 1811), el fundador de la institución Schnepfenthal, una escuela dedicada a las nuevos métodos educativos, derivados en gran parte de las ideas de Rousseau. Ya en los “Pensamientos azarosos en una noche de insomnio" de 1788 se menciona la obra de Pestalozzi Lienhard und Gertrud (Sch. 7, I, p. 11 / GA II, 1, p. 104). Y en 1793 realiza en compañía de sus amigos el poeta Baggesen y el crítico de arte Fernow una visita al gran educador suizo, quien, durante una extensa conversación, les detalló a los invitados el método que había creado ${ }^{4}$. Fichte, además, le insistió en la necesidad de dar a conocer sus estudios sobre la naturaleza humana y su formación progresiva, cuyo resultado fue la publicación en 1797 de Mi indagación sobre el curso de la naturaleza en el desarrollo humano. No obstante, el interés de Fichte por la pedagogía de Pestalozzi se mantuvo latente situado en un segundo plano, ya que por entonces el idealista se encontraba más ocupado en construir su propia filosofía, hasta que volvió a activarse en la madurez, cuando comprendió la necesidad urgente de consolidar una educación popular y pública, que sirviese de instrumento para la regeneración de una Alemania desmembrada y vencida. De hecho, en 1807 pregunta por carta a su mujer si ha leído Cómo Gertrudis educa a sus hijos, libro que Pestalozzi dio a la luz pública en 1801, y le informa de que en ese momento se encuentra estudiando otra obra de él de reciente publicación: Un vistazo sobre mis fines y experimentos educativos. En esa misma carta Fichte reconoce que el sistema educativo del suizo le parece "el verdadero remedio santo para la humanidad enferma, así como el único medio de hacer entender de forma adecuada la Doctrina de la ciencia”. ${ }^{5}$

Sin embargo, no se puede negar que para el Fichte de Jena la educación ya era el único medio que permite ingresar al mundo humano, a pesar de que no abordara el tema de manera directa, quitando las Lecciones sobre la destinación del sabio del 94. Por ejemplo, si nos atenemos al Parágrafo VI de la Fundamentación del derecho natural, donde se ponen las bases para una teoría de la intersubjetividad, se observa que, dada la indeterminación completa con que el hombre llega a la vida, únicamente puede perseverar en ella y encauzarse hacia la humanidad, es decir, hacia el pensamiento, el lenguaje, el arte o las acciones morales, mediante una apelación amorosa por parte de otro individuo que se traduce en unos cuidados y en una enseñanza, la cual consiste básicamente en dejar un espacio para que el otro pueda manifestarse, en respetarlo incluso antes de que sea capaz de desarrollar sus facultades racionales, dándole la oportunidad de actuar sin coacciones, sintiéndose libre. Este reino humano es, por oposición a la naturaleza, que está determinada esencialmente por la repetición y el instinto, un mundo cultural, cuya entrada sólo puede franquearse previo aprendizaje de ciertos códigos.

4 Cfr. Léon, 1922-1927 [1954-59], pp. 211-214.

5 Carta del 3 de junio de 1807, Sch. 550, II, pp. 454 s. / GA III, 6, p. 121. 
Más adelante, en los Aforismos sobre la educación, un texto escrito por Fichte en 1804 a fin de justificar un proyecto pedagógico que finalmente no se llevó a cabo, concretamente, un plan para educar junto a los hijos de otras familias al suyo propio, Immanuel Hermann, quien entonces tenía ocho años, el filósofo deja bien en claro los objetivos de la educación. Y en un estilo que recuerda al inicio del artículo kantiano sobre ¿Qué es la ilustración?, explica que esto significa convertir al alumno en maestro, conseguir que se empodere y se haga dueño de sí mismo, del conjunto de sus capacidades. Sólo una educación integral, no parcializada, permite producir el saber desde sí: crear conocimientos, descubrir, inventar y también tomar las decisiones pertinentes para su aplicación. Se trata de que el educando se vuelva un ser autónomo y eso se logra cuando se elude hacer especialistas que funcionen como engranajes ciegos de una máquina cuya estructura completa y, por tanto, su fin general, desconocen. Se consigue cuando el proceso educativo no consiste en un adoctrinamiento, en una imposición que se irradia desde fuera hacia dentro como si se tratara de "ciencia infusa", generando lo que Fichte llama en Sobre la esencia del sabio de 1805, “ "ineptos integrales", esto es, individuos incapaces de subordinar a su libertad los fragmentos dispersos de su formación y de hacerla crecer orgánicamente, per intus susceptionem, según el modelo ofrecido por Kant para la razón (KrV A 833/B 861). En la búsqueda de una completitud armónica entre las distintas esferas del espíritu, Fichte coincide no sólo con las ideas de Pestalozzi sino también con el objetivo de Schiller en sus Cartas sobre la educación estética del hombre. Detengámonos un momento en la lectura del primer aforismo para comprobar hasta qué punto esta educación para la libertad rechaza los objetivos perseguidos por la enseñanza actual y concuerda, a su vez, con los planteamientos que de la cultura y la sabiduría hacía Fichte desde su juventud:

Educar a un hombre significa darle la oportunidad de hacerse por sí mismo maestro y autodominador absoluto de toda su fuerza. De toda su fuerza, digo: pues la fuerza del hombre es una y es un todo unificado. Tener a la vista como finalidad, ya durante la educación, un uso aislado de esta fuerza -o, según se ha designado, educar al discípulo para una profesión- sería sólo superficial, si es que no perjudicial. Empequeñece la fuerza y la hace esclava de la profesión pretendida, debiendo ser ella la dominadora de ésta. A la fuerza que se ha formado entera y armónicamente se le puede confiar el aspecto por el cual quiera ella aproximarse al mundo y a la praxis en él; o bien, en toda profesión, no se trata de para qué se ha sido educado ni de qué se ha aprendido, sino de qué se es. Quien ya es efectivamente un ser racional y en todo momento activo por sí mismo, fácilmente se hará en aquello que en su situación deba ser. Pero quien mediante alguna suerte de ejercitación (Dressur = amaestramiento) ha reemplazado el instinto animal, que, por desgracia, es menesteroso, queda justamente atrapado en este límite que lo circunda como una segunda naturaleza impenetrable para él, y la educación e instrucción lo han limitado y matado en lugar de liberarlo y haberlo capacitado para un crecimiento viviente desde sí mismo. ${ }^{7}$

6 Ueber das Wesen des Gelehrten und seine Erscheinungen im Gebiete der Freiheit (en adelante UWG), GA I, 8, p. 67. El término original es Stümper.

7 Aphorismen über Erziehung (en adelante AuE), GA II 7, 17. 
De acuerdo con el texto, la educación opera sobre "toda la fuerza del hombre". El término que utiliza Fichte aquí es Kraft. Y esta fuerza es en su núcleo pura espontaneidad espiritual. Representa la absoluta actividad de autoconstitución, la presencia de la Thathandlung en lo finito bajo la forma de una tendencia que, al encontrar sus límites, se convierte en impulso (Trieb), y a la cual en este momento Fichte denomina vida divina, por no ser empírica y finita. Dado que éste es su punto de partida, el proceso educativo se contradice con cualquier ejercicio de domesticación y adiestramiento que intente forzar desde fuera la comprensión o la voluntad. De hecho, las reglas o las prescripciones externas suelen aceptarse por miedo, conveniencia o comodidad, de modo que generan esclavitud mental e hipocresía social. Ellas sólo adquieren verdadero significado cuando se admiten por propia convicción, es decir, si son el resultado de un procedimiento interior que se produce al nivel de la esencia (Wesen) y luego se manifiesta en el ámbito de la libertad, de la transformación del mundo. Como resultado, la educación no tiene que ver con aquello que se tiene o se ha aprendido sino con lo que se es. Si precisamente sirve para apuntalar y cimentar cualquier transformación política previniendo su regresión, es porque genera una actitud inmodificable, a prueba de las modas y las variables circunstancias. Así, dice Fichte en Los Discursos a la nación alemana:

Toda formación aspira a la producción de un ser (Seyn) firme, determinado y perseverante, que no se transforme, sino que es y no puede ser de otro modo que como es. Si no aspirara a tal ser, no sería formación sino un juego inútil. ${ }^{8}$

Dos consecuencias, al menos, pueden extraerse del carácter espontáneo e interior que Fichte atribuye a la fuerza, a la actividad humana. Primero, ésa es la razón de su admiración por los sistemas educativos que apelan al desarrollo natural del aprendizaje y abren gradualmente el acceso a los distintos niveles de conocimientos y actitudes en función de las necesidades y los sentimientos propios del educando, como ocurre, por ejemplo, en Rousseau o en Pestalozzi. Segundo, la educación tiene que apoyarse en lo ya existente y le es imposible cambiar al hombre de raíz. Semejante principio asegura la completa inviolabilidad de la libertad del educando, porque el maestro sólo podrá limitarse a hacer patente y a fortalecer algo que ya estaba palpitando en el individuo. El uso de la coacción como técnica educativa no está permitido, ya no porque contradiga su meta: la liberación de la humanidad, sino por ser un medio absurdo e ineficaz. Continúa Fichte en Los discursos a la nación alemana:

La voluntad del hombre ya tiene fija su dirección antes de la amonestación e independientemente de ella; si coincide con la amonestación, llega demasiado tarde y sin ella el hombre hubiera hecho también lo mismo que le amonestas; si está en contradicción, lo máximo que lograrás es aturdirlo por unos instantes; tan pronto

8 Reden an die deutsche Nation, (en adelante RDN), GA I, 10, pp. $117 \mathrm{~s}$. 
llegue la ocasión, se olvidará de sí mismo y de tu amonestación y seguirá su inclinación natural. Si quieres inducirlo a algo, tienes que hacer más que simplemente hablarle: has de formarlo, hacerlo de tal manera que no pueda querer de modo distinto del que tú quieras que él quiera (RDN, GA I, 10, p. 118).

Pero no nos equivoquemos. No sólo la persuasión es inútil. También lo es cualquier otra técnica de sugestión que busque subordinar o someter al educando a los intereses del educador. La puesta en marcha de la voluntad siempre procede con total independencia de los objetos y los acontecimientos empíricos. Si su funcionamiento obedeciera a algo más que sus propias leyes, no podrían sostenerse ni la responsabilidad ni la libertad humanas. Esta completa autonomía de la voluntad, intrínseca al pensamiento ético kantiano, adquiere en Fichte importantes consecuencias. Así, en las lecciones sobre el destino del sabio de 1794, defiende la anterioridad de todo impulso respecto del objeto al cual se dirige e insiste hasta tal punto en el tema que en la edición danesa de la obra agrega una nota sobre el asunto. Allí sostiene que el objeto es elegido por el sujeto antes de que pueda ofrecérsele tentadoramente, por ello, toda acción es, en sentido estricto, una creación que se fragua desde dentro. El impulso, raíz de la voluntad, aspira necesariamente al bien -digamos a su bien- so pena de contradecirse y aniquilarse a sí mismo. Y, por tanto, actuar, ser y bien coinciden. Dado que el fin de la voluntad no es empírico, los ideales humanos no dependen de las circunstancias y, por tanto, existe en los individuos un núcleo inmodificable, aunque no en el mismo grado de pureza. Precisamente, ésta es la tarea de la educación: dejar surgir el impulso originario hacia el bien, idéntico en todos, elevar a los hombres y de esta manera nivelarlos entre sí. Para ello intentará formar en los educandos una voluntad infalible, sobrepuesta a las inclinaciones, que jamás podrá constituirse mediante la simple imitación, aceptando el ejemplo sin elaborarlo, sino apropiándose de él mediante la reflexión que surge del ejercicio repetido gracias al libre juego de acciones dentro de la comunidad. De esta manera, el nuevo contenido terminará por incorporarse a esa totalidad orgánica que lo precedía. ${ }^{10}$

Si no tenemos en cuenta esta teoría, así como la distinción entre libertad y libre albedrío, podrían resultarnos paradójicas ciertas afirmaciones de Fichte, como la siguiente - que, por cierto, pertenece también a Los discursos a la nación alemana:

9 Einige Vorlesungen über die Bestimmung des Gelehrten (en adelante UBG), GA I, 3, pp $31 \mathrm{~s}$. Cfr. Nota a la edición danesa de 1796 en las obras de Fichte publicadas por Fritz Medicus, vol. I, 214 b. Cito esta edición, pues en ella la nota aparece en alemán y no en su idioma original, como sí ocurre en la Gesamtausgabe (GA I, 3, pp. 73 s.): "En el [impulso] está previamente determinado lo que puede y nos hará felices. [...] Así sucede con todos los impulsos del hombre: tanto con los físicos como con los morales. Siento placer al comer y beber no porque un par de veces me haya gustado. De dónde podría haber venido el que me hubieran gustado, sino de que, prescindiendo [del hecho] de [haber] comido o [haber] bebido, siento placer de alimentarme de una determinada manera".

10 Ueber den Unterschied zwishen Geist und Buchstaben in der Philosophie, GA II, 3 pp. 338-342. 162-165. 
En reconocer y en contar con un libre albedrío del educando, radica el primer error de la presente educación, así como la clara confesión de su impotencia y nulidad.

Por el contrario, la nueva educación debería consistir, precisamente, en aniquilar de raíz la libertad de la voluntad de cuyo cultivo se hace cargo, produciendo en ella, en cambio, una rigurosa necesidad de las decisiones y la imposibilidad de lo puesto, con la que luego se podría contar y confiar con seguridad (RDN, GA I, 10, pp.117 s).

En el libre albedrío, en cuanto capacidad de opción que permite escoger entre lo bueno y lo malo, los objetos a elegir son previos a la decisión de la voluntad. En consecuencia, constituye una manifestación muy limitada de la libertad, porque en semejante elección el sujeto depende de las circunstancias que se le presentan. Este condicionamiento exterior hace que la voluntad se vuelva vacilante y someta sus decisiones a la valoración de los resultados que ellas pudieran acarrear, o de otros intereses completamente extraños a la moralidad. Y esto es algo que ya Fichte había denunciado desde la misma Reseña de Enesidemo, al señalar que la categoría de posibilidad pertenece al ámbito de la razón teórica y no de la práctica (GA I, 2 , pp. $388 \mathrm{~s}$ ). De hecho, el ejercicio del libre albedrío puede conducir a la realización de acciones contrarias a la ley moral, por ejemplo, cuando, entre las múltiples alternativas que se ofrecen, se elige el mal menor. A causa de ello, el objetivo del educador no ha de ser el fomento del libre albedrío, porque provoca la indecisión, la perplejidad y puede conducir a una decisión infame. Pero tampoco ha de querer inculcar en el educando unos principios que le sean ajenos y que, por tanto, jamás podría llegar a asimilar. Su misión es despertar el automatismo propio de la voluntad que acabamos de describir, basado en la aspiración absoluta de afirmación y de armonía consigo misma, que indefectiblemente culmina con un acto de aplicación de la ley moral ${ }^{11}$. Y como dicha ley es una norma de la razón y, por tanto, común a todos los individuos, la decisión del educando coincide con aquello que quería el maestro. La educación, pues, es eminentemente ética y ha de guiar al alumno hacia la máxima libertad, que no es otra cosa que necesidad interna. Dice Fichte en Los discursos a la nación alemana:

La esencia propia de [...] la nueva educación [...] consiste en ser el arte [Kunst] prudente [besonnene] y seguro de formar al alumno para la pura eticidad [Sittlichkeit] (RDN, GAI, 10, p. 131).

Educar es un arte, no una ciencia. Como tal, requiere prudencia, capacidad para aplicar la sabiduría a situaciones empíricas concretas, a individuos siempre distintos, rodeados de entornos nunca del todo coincidentes. Por eso, en las lecciones del 94, Fichte exige al sabio, en cuanto maestro de la humanidad, tres tipos de conocimiento:

11 En Algunas lecciones del destino del sabio Fichte enuncia el imperativo categórico como ley de coherencia o no contradicción consigo mismo: "Obra de tal modo que la máxima de tus acciones pueda convertirse en ley constante de tu voluntad” (UBG, GA I, 3, p. 30). 
racional y filosófico, es decir, de las aptitudes y necesidades del hombre; empírico, de los medios para satisfacer dichas necesidades; e histórico-filosófico de la sociedad, el cual permite establecer en qué grado de desarrollo ésta se encuentra y calcular en cada caso el posible progreso del género humano (UBG, GA I, 3, pp. 52 s'. El objetivo de la cultura, así como el de la educación, es hacer retroceder los límites del Noyo, de todo aquello que impide la expansión del Yo. Se trata -como leímos en el primer aforismo- de transformar el mundo, por tanto, el fin de la educación siempre es práctico. Y dado que el Yo del que hablamos es intersubjetivo y se va formando históricamente en la relación que los individuos establecen entre sí, su esencia es ética y los medios para realizar esa transformación en el mundo real -según se indica en Sobre la esencia del sabio- son el derecho y la belleza (UWG, GA I, 8, p. 66). Eso descarta que la pedagogía propugnada sea moralizante. El educador puede declarar de vez en cuando la ley moral, por ejemplo, "no mentir bajo ningún concepto, no hablar ni actuar conscientemente y a sabiendas contra la propia conciencia" (AuE, GA II, 7, p. 21), pero ha de abstenerse de ofrecer una instrucción moral positiva. Dice Fichte en el noveno de sus Aforismos:

Una educación moral positiva, es decir, una educación tal que se proponga como fin, y lo exprese de modo explícito, formar al discípulo para la virtud, no la hay. Más bien, tal proceder mataría el sentido moral interno y formaría sólo hipócritas y mojigatos sin carácter (ídem).

Lo mismo vale para la religión, siendo indiferente para el educador de qué credo se trate. En cualquier caso, el discípulo habrá de procurársela por su cuenta mediante un sacerdote, cuando tenga edad suficiente para participar en los misterios (AuE, Aforismo 10, GA II, 7, p. 21). La única propuesta concreta que hace Fichte en los Aforismos consiste en dar una rotunda prioridad al estudio de las lenguas clásicas, en particular, al griego, que, a pesar de tener un parentesco estructural con el alemán, fomentaría la flexibilidad conceptual por contraste, ya que implica una expresión, una imagen distinta del mundo (AuE, Aforismos 2 y 3, GA II, 7, p. 17 s). A partir de la enseñanza gramatical que aportan las lenguas antiguas, pueden aprenderse fácilmente las modernas, cuyo conocimiento es proporcionado en la vida inmediata (AuE, Aforismo 5, GA II, 7, p. 19). Lo mismo puede decirse del mundo circundante, que ha de ser procurado mediante la propia experiencia o escuchando conversaciones. Fichte sólo recomienda el estudio de la antigüedad: su historia, su geografía, sus ideas (AuE, Aforismo 4, GA II, 7, p. 18). En cuanto a las ciencias, prefiere que primero se fortalezca la fantasía haciéndose libre y autónoma, para que, una vez comprobado el poder constructor que el hombre tiene a través de la imagen, el alumno emprenda el aprendizaje de esas reglas científicas, universales pero también subjetivas, que rigen la naturaleza. Como resultado, considera perjudicial el estudio de la física antes de cierta edad, puesto que anquilosa la imaginación, que, en definitiva, es la 
facultad que construye el mundo. Por eso, sólo aconseja en los niños el aprendizaje de la matemática, fundamental para la maduración de las categorías lógicas (AuE, Aforismo 6, GA II 7, pp. 19 s.).

Aparte de esta disciplina, es evidente que le concede una preeminencia a las lenguas clásicas y a las humanidades desde el comienzo de la vida educativa del niño y que hace de ellas el pilar para el desarrollo de la libertad y la creatividad. No deja de ser curioso comprobar que éstas son, justamente, las disciplinas que en los últimos años han sido despreciadas por nuestra sociedad por inútiles, y notablemente reducidas cuando no desterradas en los programas escolares a raíz de las sucesivas reformas pedagógicas. La intención que se esconde tras el rechazo de las humanidades en la sociedad actual constituye el reverso de lo que anima su reivindicación en nuestro filósofo. Fichte pretende defender una educación civil al servicio del conjunto de la sociedad, ajena al materialismo y al egoísmo. De este modo, piensa la cultura como un nivelador social que elimina la discriminación introducida por la naturaleza aleatoriamente según el principio de diversidad, al distribuir las capacidades de una manera poco armoniosa entre la población. La instrucción, entonces, se convierte en medio para remontar las diferencias sociales (UBG, GA I, 3, pp. 44 s). Por eso, las propuestas pedagógicas de Fichte evitan entrar en ideologías preconcebidas, partidistas o interesadas. Aspiran a la sabiduría y apuntan a la verdad, que es la fuente misma de la vida. De ahí que no sólo coincidan con los ideales educativos de las escuelas populares de Pestalozzi sino, en el caso de España, con los de la Institución Libre de Enseñanza, inspirada por el pensamiento de Krause, quien precisamente fue discípulo del idealista. Sorprendente es, además, la coincidencia con las escuelas Waldorf, fundadas por el creador de la antroposofía, Rudolf Steiner, cuya vida espiritual dio un completo viraje al leer a Fichte, autor sobre quien escribió su tesis doctoral. Su pedagogía es presentada por sus seguidores como una educación para la libertad cuyos objetivos fueron expresados por él en términos muy parecidos a los que habría usado Fichte:

Vivir en el amor por la acción y dejar vivir por la comprensión de la voluntad ajena, ésta es la máxima fundamental del hombre libre" -dice en La filosofía de la libertad (el título lo dice todo) (Steiner, 2002).

Aunque la educación idealista sea definida como esencialmente espiritual, su carácter integrador incluye también al cuerpo, igual que ocurre en los tres ejemplos concordantes que acabamos de mencionar. Ya desde las lecciones del 94, Fichte había admitido que la tarea de la cultura incluye el perfeccionamiento de la sensibilidad y que éste es el más alto empleo al que se la puede destinar (UBG, GA I, 3, p. 31). De acuerdo con esto, en la Fundamentación del derecho natural, había concebido el cuerpo como una prolongación material del Yo, el único campo donde éste puede 
decidir legítimamente con total libertad y, por tanto, como un instrumento capaz de transformar el mundo ${ }^{12}$. En el quinto Aforismo avanza un paso más y establece cuáles son las disciplinas que sirven para el desarrollo de los sentidos, pero también para su fijación, ya que la sensibilidad tiende necesariamente a la dispersión. Así, además de aconsejar convertir la concentración en un hábito y desdeñar cualquier distracción, recomienda el disfrute del aire libre y ejercicios gimnásticos como el baile, la lucha deportiva, la esgrima o la hípica, "orientados en su conjunto a la finalidad de traer el cuerpo bajo el dominio del espíritu y convertirlo de este modo en un instrumento fuerte y resistente" (AuE, Aforismo 8, GA II, 7, p. 20).

Para la constitución de la nueva escuela, Fichte también aporta algunas sugerencias en el noveno y el décimo de Los discursos a la nación alemana, si bien en su mayor parte se limita a sentar las bases filosóficas sin descender al detalle. En los aspectos prácticos remite a Pestalozzi, cuyo método renovador él mismo había introducido en Berlín. Sin embargo, existen divergencias con el educador suizo, por ejemplo, respecto de la valoración excesiva que éste hace del aprendizaje de la lectura y la escritura, o de la gran importancia que otorga a los trabajos manuales, a los cuales el filósofo considera meramente accesorios. Por otra parte, Fichte, matiza notablemente la intervención de la familia en los primeros tramos de la educación y, más concretamente, de la madre en la formación del niño, a la cual Pestalozzi otorgaba un papel preponderante. Él, en cambio, desconfía de ambos y prefiere poner la educación en manos de profesionales (AuE, Aforismo 11, GA II, 7, pp. 21 s), sentando así las bases para una enseñanza pública nacional. Precisamente, la misma que cuestiona el capitalismo neoliberal, a pesar de que ofrezca una solución eficaz, por ejemplo, al tema de la educación sexual, la pedofilia y los abusos infantiles o de adolescentes, que, en su mayoría, se dan en el seno de la familia, cuando no en el ámbito de la iglesia. En este contexto, no puedo dejar de elogiar la denuncia que Fichte hace sobre la tolerancia excesiva que caracteriza a la edad moderna con su permanente crítica al rigor y al esfuerzo'13, esa comodidad del "todo fácil”, del "no me pongan límites y obstáculos", de ese lema que define al hombre-masa orteguiano: "todo son derechos y ninguna obligación", completamente aplicable a lo que ocurre hoy en día en los centros escolares de todo el mundo. La intervención de las familias en la escuela se ejerce siempre con la excusa de proteger a los educandos y asegurar la igualdad de oportunidades. Pero las aspiraciones personales de los padres y la

12 Grundlage des Naturrechts nach der Prinzipien der Wissenschaftslehre, Parágrafos V y VI. Véase asimismo Ueber den Unterschied zwishen Geist und Buchstaben in der Philosophie, GA II, 3, pp. $319 \mathrm{~s}$.

13 En Los caracteres de la época actual Fichte define su propia época como la caída de la humanidad en estado de plena pecaminosidad a causa del materialismo, la mediocridad y la crítica al esfuerzo (Grundzüge des gegenwärtigen Zeitalters (en adelante GgZA) GA I, 8, pp. 206 s.). También se refiere a este último asunto en Sobre la esencia del sabio (GA I, 8 p.67, pp. 69 s.), así como a la pereza y a la indolencia del estudiante en las pp. $99 \mathrm{~s}$. 
ignorancia en temas -como los pedagógicos- donde todo el mundo cree poder opinar, consiguen que esta reivindicación encubra muchas veces el narcisismo paterno, el deseo de que los hijos propios sean considerados y reconocidos sin más como los mejores. Por este motivo, la intromisión familiar indiscriminada en la escuela ha sido uno de los factores que más depauperaron la educación en los últimos años, produciendo una caída generalizada en la mediocridad e introduciendo la violencia hacia el profesor en las aulas, cuya autoridad para juzgar o valorar al alumno es puesta en duda por los padres.

Volviendo al tema de las diferencias entre los dos autores, probablemente tengan que ver con la experiencia personal de cada uno y el campo al que dedicaron sus esfuerzos transformadores. En el caso de Pestalozzi, hijo de un oftalmólogo pero huérfano desde niño y educado por su madre, es importante destacar el rotundo éxito de su proyecto de fundar escuelas con el fin de erradicar el analfabetismo. En su tentativa de eliminar la pobreza, creó primero una escuela-granja y, tras su fracaso, fundó una escuela-taller o una escuela industrial. Fichte, en cambio, fue instruido fuera del entorno familiar desde los ocho años y sus teorías pedagógicas están vinculadas directamente a su visión de la filosofía y a su práctica como preceptor y profesor universitario. En consonancia con los principios de su doctrina de la intersubjetividad, apoya la convivencia de los educandos en comunidades completamente autárquicas, en las cuales se fabriquen todos los productos consumidos y donde cada individuo trabaje, además de estudiar, en beneficio de la totalidad del grupo educativo. En el fondo, éste siempre es considerado como si fuera un reflejo en pequeño del Estado ideal, socialista y cerrado comercialmente, que propuso en su obra homónima de 1800. En tal sentido, puede decirse que Fichte tiene siempre en mente el plan de una formación política y la aspiración de que los ciudadanos alcancen una libertad que se adquiere y se ejercita en el seno de la comunidad, lo cual exige que se hagan conscientes de los deberes para con ella poniendo en práctica sus ideas.

Sobre todo en el caso de la Universidad, se ha de seguir el principio de la autonomía, basado en la autosuficiencia del saber, lo que permite tomar distancia frente a las presiones sociales y los intereses pragmáticos. Como consecuencia, la plena libertad académica afecta a la Universidad en los dos estamentos que la configuran. Por una parte, los estudiantes, en cuanto que se encuentran todavía en proceso de formación, han de gozar de un amplio espacio para probar sus ideas en el marco de una vida cooperativa, confrontándolas con las de los demás miembros de la comunidad universitaria. Como dice Fichte en Sobre la esencia del sabio:

Que escojan libremente el bien o el mal; el tiempo del estudio es sólo su tiempo de prueba. El tiempo de la decisión de su destino viene después, y la ventaja de esta organización es que el no apto aparece claramente como no apto, y ya no puede ocultarlo más (UWG, GA I, 8, p. 110). 
Por otra parte, los profesores tienen el máximo compromiso con el saber, por tanto, su prioridad es generarlo y dar cuenta de ello en sus clases, eludiendo en lo posible el libro de texto. Dicho principio dará sus máximos frutos en la posterior Universidad alemana que, según el modelo diseñado por Humboldt para Berlín -por cierto, notablemente influido por Fichte- consiste en la unión de docencia e investigación. Semejante asociación producirá un apogeo de la cultura científica y humanística en los tiempos universitarios inmediatamente posteriores, si bien hace tiempo que se encuentra en declive.

Precisamente por eso, no se puede dejar de citar la invectiva de la última lección Sobre la esencia del sabio contra la degradación de los escritores, esos maestros que no adoctrinan oralmente sino mediante la pluma, porque, si bien Fichte se refiere a sus contemporáneos, está claro que su vigencia ha aumentado hoy en día. Se trata de una denuncia de esa mayoría que escribe para hacer crecer su currículum con nuevas publicaciones y alimentar la propia soberbia con indiferencia del contenido escrito. Lo mismo podría argumentarse contra la proliferación de recensiones y estudios sobre trabajos ajenos en detrimento de la creación original. Ambas críticas son especialmente aplicables a los profesores universitarios, cuya investigación se ha convertido actualmente en una exigencia perentoria que incide en el sueldo y se comprueba al peso, de acuerdo con el modelo norteamericano, atendiendo a la cantidad de "papers", de artículos editados y expuestos en congresos, lo cual -dicho sea de paso- ha condenado a muerte al género del gran ensayo, porque -como dice Fichte- quienes levantan el peor testimonio contra los escritores son ellos mismos con sus propias obras (UWG, GA I, 8, p. 113).

En definitiva, la realización de la libertad plena en una comunidad cerrada será asumida al nivel de la instrucción superior por Humboldt. Es el principio de Einsamkeit, de soledad o aislamiento, que coincide con esa necesidad de concentración de la que habla Fichte, necesaria para fomentar el ejercicio del pensamiento y que hoy, sobre todo en su versión económica y administrativa, conocemos como autonomía universitaria, el mismo principio que ha regido hasta ahora el prestigioso desarrollo de las Universidades alemanas, así como de otras muchas instituciones educativas que hoy consideramos punteras en el mundo.

Éstos son los lineamientos fundamentales que la educación para la libertad tiene en el Fichte maduro. El proyecto, sin embargo, no funcionaría sin la figura del sabio. Las instituciones en su conjunto sólo poseen un carácter instrumental y, por tanto, amoral. De ellas sólo puede predicarse la efectividad, es decir, su adecuación para un cierto fin, que sí es susceptible de ser juzgado éticamente. Pero como los fines más altos sólo pueden ser alcanzados por hombres buenos, todo el funcionamiento de la sociedad se hace depender de la bondad de uno o varios individuos que la conducen, del carácter mesiánico del sabio. $Y$ con ello se sienta el 
principio antimaquiavélico de que no importan los métodos sino los hombres que los aplican y los objetivos que con ello persiguen. Dice Fichte en las lecciones de 1805:

Es además claro que el sabio, en este sentido en el que hace absolutamente todo lo posible para resolver la pugna entre la pureza interior de la idea y su eficiencia externa, queda asignado exclusivamente a su propia buena voluntad, y acerca de esto no tiene otro juez que él mismo, ni ningún otro estímulo más que en sí mismo. Acerca de esto no puede juzgarle ningún otro; ni siquiera puede nadie comprenderle aquí del todo, ni adivinar la más profunda intención de su comportamiento. Bien lejos de que el respeto por el juicio ajeno pueda apoyar en esta región su propia buena voluntad, aquí tiene que estar incluso más allá del juicio ajeno, y considerarlo como si no lo hubiera en absoluto. Queda entregado a su buena voluntad enérgica inconmovible frente a las tentaciones de impulsos muy nobles [... como] exponer vulgarmente lo sagrado para que llegue a la comunidad, y de este modo profanarlo [...] o desdeñar enteramente a su época, renunciar a ella y no querer tener nada que ver con ella (UWG, GA I, 8, p. 108).

Todo reposa en el compromiso total del sabio con la humanidad. Todo depende de su integridad y honestidad, lo cual, sin duda, recuerda a la situación del reconocimiento intersubjetivo en la Fundamentación del derecho natural, cuando Fichte afirma: “Mi racionalidad depende, según esto, del libre albedrío, de la buena voluntad de otro" (GNR, GA I, 3, p. 375).

Sin embargo, el hecho de que se trate de un acto voluntario no significa en modo alguno que el reconocimiento sea el resultado de un puro altruismo, ya que la aceptación del otro como fin en sí mismo, es decir, como individuo capaz de decidir libremente, no sólo representa para él la oportunidad de poner en marcha sus facultades humanas sino que revierte sobre el primer sujeto permitiéndole reconocerse a sí mismo como un ser racional y asumir responsablemente su propia libertad. Lo mismo ocurre con el sabio, quien, como consecuencia, ha de ser el mejor hombre de su generación, un representante de la humanidad toda en su más alto grado de perfección, según se sostiene ya en las lecciones del $94^{14}$.

En realidad, desde entonces -desde esas lecciones-, Fichte no modificó sustancialmente el papel del sabio. Su función sigue siendo política: vigilar y fomentar el progreso armónico de todas las clases sociales (UBG, GA I, 3, p. 54), ${ }^{15}$ establecidas gracias a una racional división del trabajo. Pero en su etapa de madurez lo perfila algo más, distinguiendo tres aspectos que pueden aparecer conjuntamente en una misma persona -y entonces estaríamos ante la presencia del más elevado y completo exponente del sabio-, o pueden hallarse disociadas, dando lugar a tres tipos diferentes: los gobernantes (Regenten), que actuarían como reyes o consejeros del rey, los educadores y los escritores, encargados ambos de comunicar la sabiduría,

14 UBG, GA I, 3, p. 58. Véase también UWG, GA I, 8, p. 110.

15 Sobre el tema del progreso, véase System der Sittenlehre, GA I, 5, p. 141. 
aunque a través de distintos medios. ${ }^{16}$ Además, el sabio se revela ahora como una encarnación de lo absoluto y un canal de comunicación con él. Es un testimonio viviente de la vida divina que se desborda en ideas (Gedanken). Estas ideas poseen una esencia espiritual y se captan intuitiva y emocionalmente, por eso la obligación social que impele al sabio a conducir y enseñar a los hombres se presenta aquí como un producto del amor a las ideas (UWG, GA I, 8, pp. 67 s). Y esto no es del todo nuevo, no es el resultado de una conciencia maliciosa tras una acusación de ateísmo, porque Fichte siempre había reconocido que la misión del sabio es puramente vocacional y representa un compromiso sagrado. No en vano Jacobi lo llamó "Mesías de la razón especulativa" ${ }^{17}$ y él mismo se había definido no sólo como un soldado sino como un “sacerdote de la verdad" (UBG, GA I, 3, pp. 58) dispuesto a dar su vida por ella. Lo divino tampoco tiene por qué sustancializarse ni pensarse como una persona, puede ser el fluir de la vida que, al final, termina ordenándose a través de relaciones morales.

Pero lo que sí ha cambiado, y radicalmente, es el contexto en que se desenvuelve el sabio, la propuesta política en la cual se enmarca su labor, que no es otra que la de un Estado hiperestructurado y clausurado sobre sí mismo como el que Fichte diseña en 1800. Se trata de una dictadura, una tiranía estatal, por mucho que se la haya querido suavizar destacando la importancia que en ella adquieren la dignidad personal y el ocio ${ }^{18}$ o refiriéndose a ella con el eufemismo de “dictadura educativa" ${ }_{19}$, término con el que seguramente se la ha querido parangonar a la república platónica. Tampoco puede decirse que este tipo de Estado sea capaz de salvaguardar, mucho menos de potenciar, la individualidad. Su cerrazón interna y externa hacen de él una máquina cuyos engranajes son los ciudadanos mismos y no un organismo que vive en y a través de cada uno de sus miembros. ${ }^{20} \mathrm{Y}$, sin embargo, en Los caracteres de

16 La distinción es enunciada en Sobre la esencia del sabio, GA I, 8, pp. 114 s., pero a cada una de estas clases Fichte dedica un capítulo de su obra.

17 Carta a Fichte del 3 de marzo de 1799. Sch. 346, II, p. 26 / GA III, 3, p. 226. Expresiones similares: "rey de los judíos de la razón especulativa" (ídem, p. 227), o "judío de la razón especulativa" (ídem, p. 228).

18 "Su sistema sustituirá este orden general en el que la fortuna de unos es la miseria de los demás por una ley nueva, de acuerdo con la cual el Estado se reserva la tarea de aumentar la justicia; pero la violencia estatal carecerá de sentido si no se permite a su vez que el ciudadano tenga dignidad espiritual y ejerza funciones que hagan verdaderamente de él un hombre. De ahí la importancia que da Fichte al concepto de ocio. En todo el conjunto de su obra, aparece como preocupación central, en medio de las violencias que impone un orden nuevo, el respetar y promover la libertad humana" (Droz, 1984, p. 562-564).

19 Véase, p.e., Der transzendentale Gedanke, pp. 201 y 203.

20 A finales del siglo XVIII se utilizó la expresión "Estado-máquina” para referirse al Estado absolutista, que impone sus leyes automáticamente a los ciudadanos sin contar con ellos. Fr. Schiller rechazó esta clase de Estado por su carácter inhumano en las Cartas sobre la educación estética del hombre. Siguiendo sus sugerencias, los románticos propusieron un Estado orgánico, donde los ciudadanos no son medios sino que participan de la finalidad del todo. Cf. Sánchez Meca, 2013, cap. 2.1. 
la época actual, obra contemporánea a las que estamos analizando, Fichte supone ingenuamente que esta clase de Estado, el racional, es meramente provisorio, una ortopedia, un instrumento que, una vez que corrige, está condenado a desaparecer:

Según nosotros, el Estado absoluto es, en cuanto a su forma, una institución artificiosa para dirigir todas las fuerzas individuales a la vida de la especie y fundirlas en esta vida para realizar y exteriorizar en los individuos la forma de la idea en general, suficientemente descrita con anterioridad. Como para ello no se cuenta con la vida interior y la actividad originarias de la idea en los espíritus de los hombres [...], como más bien esta institución opera desde fuera sobre los individuos, que no sienten ningún placer, sino más bien repugnancia, en sacrificar su vida individual a la especie, se comprende que esta institución resulte una institución coactiva. Para aquellos individuos a los cuales las ideas hubiesen alcanzado vida interior propia y no quisiesen ni deseasen absolutamente ninguna otra cosa que sacrificar su vida a la especie, no se necesitaría de la coacción; ésta desaparecería para ellos, y el Estado se reduciría, con respecto a ellos, a aquella unidad que abarcase el todo, aclararía e interpretaría en cada uno la finalidad primera y más próxima de la especie y situaría la fuerza servicial en su justo lugar (GgZA, GA I, 8, pp. 307 s). ${ }^{21}$

A modo de conclusión, podría decirse de la trayectoria del pensamiento político de Fichte en su conjunto algo que también es aplicable al de Platón: que refleja el esfuerzo desesperado de un hombre que cree en los ideales pero descubre a cada paso la tremenda dificultad que existe para hacerlos realidad. En las primeras obras, por ejemplo, en Algunas lecciones sobre el destino del sabio, la anarquía es lejana pero posible y se distingue perfectamente del puro ideal, porque se trata de una comunidad en la que puede haber errores e injusticias, pero en la cual se aceptará espontáneamente la enmienda de los mismos (UBG, GA I, 3, pp. 37 s). Pero en la última obra política, publicada unos meses antes de su muerte, la Doctrina del Estado, Fichte reconoce que el primer presupuesto para la constitución del reino de la razón es que sólo Dios existe (Staatslehre, GA II, 16, p. 63), postula el final de la modernidad y el inicio de una etapa histórica aún por venir, a la cual califica como "reino de los cielos" y define en términos claramente religiosos, reconociendo que ha de ser una teocracia (Staatslehre, GA II, 16, p. 164 s). Todos sabemos de la provocación del lenguaje fichteano, antes y después de la polémica del ateísmo, y que esta asociación de la que hablamos no es otra cosa que el reino de los fines, el autogobierno o la anarquía, transformada ahora en una comunidad de santos. En muchos sentidos, la propuesta de Fichte es similar a la de Joaquín de Fiore, reeditada por Lessing al final de la Educación del género humano, una obra donde la historia se presenta como una escuela donde Dios imparte sus lecciones con el fin de ayudar a la humanidad para que se eleve sobre sí misma hasta alcanzar el máximo nivel de espiritualidad. Pero, a diferencia de la comunidad política a la que el filósofo se refería en su juventud, en esta nueva versión el reino se desdibuja tras

21 Cf. Beitrag zur Berichtigung der Urtheile des Publiklums über die Französische Revolution, GA I, 1, p. 253 y UBG, GA I, 3, p. 37. 
las brumas de la utopía y culmina siendo sepultado definitivamente en el terreno de lo inalcanzable.

\section{Referencias}

Bergmann, E. (2017). Fichte, zum Deutschtum: Eine Darstellung der Fichteschen Erziehung. Bremen: Outlook Verlagsgesellschaft ( $1^{\text {a }}$ edición: 1915, Leipzig: Felix Meiner).

Droz, J. (1984). Historia general del socialismo. Madrid: Ediciones Destino.

Fichte, J. G. (1962-2012). Gesamtausgabe der Bayerischen Akademie der Wissenschaften, hrsg. von R. Lauth, H. Jacob und H. Gliwitzky, etc. Stuttgart/ Bad Cannstadt, Fromann-Holzboog (GA I: Werke; GA II: Nachgelassene Schriften; GA III: Briefwechsel; GA IV: Kollegnachschriften).

Fichte, J. G. (1925). Fichtes Briefwechsel, hrsg. von Hans Schulz. Leipzig: Haessel ( $2^{\text {a }}$ edición aumentada con un Nachtrag: Leipzig: Haessel, 1930; reimpresión: Hildesheim, G. Olms, 1967).

Hahn, K. (1969). Staat, Erziehung und Wissenschaft bei Fichte. München: C. H. Beck. Hammacher, K. (ed.) (1981). Der transzendentale Gedanke, Hamburg: F. Meiner.

Kabitz, W. (1902). Studien zur Entwicklungsgeschichte der Fichteschen Wissenschaftslehre aus der Kantischen Philosophie. Berlin: Reuther \& Reichard. Reimpresión: Darmstadt: Wissenschatliche Buchgesellschaft, 1968).

Kant, I. (1781). Kritik der reinen Vernunft (KrV). Riga: Hartknoch.

Léon, X. (1922-1927). Fichte et son temps. 3 vols. Paris: Armand Colin. (Reedición 1954-59).

López-Domínguez, V. (1995). Fichte, acción y libertad. Madrid: Ediciones Pedagógicas.

López-Domínguez, V. (2020). Fichte o el yo encarnado en un mundo intersubjetivo. México/Buenos Aires: UNAM/RAGIF.

Sánchez Meca, D. (2013). Modernidad y romanticismo: para una genealogía de la actualidad. Madrid: Tecnos.

Steiner, R. (2002). La filosofía de la libertad. Madrid: Editorial Rudolf Steiner.

Esta obra está licenciada com uma Licença Creative Commons Atribuição-NãoComercial-Compartilhalgual 4.0 Internacional.

https://creativecommons.org/licenses/by-nc-sa/4.0/

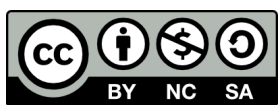

